\title{
SECTORIAL OPERATORS WITH CONVOLUTION TERM
}

\author{
Veli SHAKHMUROV AND Rishad SHAHMUROV
}

\begin{abstract}
In the present paper, separability properties of convolution-differential equations with unbounded operator coefficients in Banach valued $L_{p}$ spaces are investigated. A coercive estimate for resolvent of corresponding realization operator, especially, its $R$-sectoriality is obtained. Finally, these results applied to establish maximal regularity of Cauchy problem for the abstract parabolic convolution equations and integro-differential equations on infinite dimension state spaces.
\end{abstract}

Mathematics subject classification (2010): 34G10, 45J05, 45K05.

Keywords and phrases: Banach-valued $L_{p}$ spaces, operator-valued multipliers, abstract Cauchy problem, sectorial operators, boundary value problems, integro-differential equations.

\section{REFERENCES}

[1] H. AmAnN, Linear and quasi-linear equations 1, Birkhauser, 1995.

[2] H. Amann, Operator-valued Fourier multipliers, vector-valued Besov spaces, and applications, Math. Nachr., 186 (1997), 5-56.

[3] R. P. Agarwal, R. Bohner, V. B. Shakhmurov, Maximal regular boundary value problems in Banach-valued weighted spaces. Boundary value problems, 1 (2005), 9-42.

[4] S. AGMON, On the eigenfunctions and on the eigenvalues of general elliptic boundary value problems, Comm. Pure Appl. Math., 15 (1962), 119-147.

[5] O. V. Besov, V. P. ILIN, S. M. NiKols KiI, Integral representations of functions and embedding theorems, Moscow, 1975.

[6] D. L. BURKHOLDER, A geometrical conditions that implies the existence certain singular integral of Banach space-valued functions, Proc. conf. Harmonic analysis in honor of Antonu Zigmund, Chicago, 1981, Wads Worth, Belmont, (1983), 270-286.

[7] J. Bourgain, Some remarks on Banach spaces in which martingale differences are unconditional, Arkiv Math., 21 (1983), 163-168.

[8] G. Dore AND S. YAKubov, Semigroup estimates and non coercive boundary value problems, Semigroup Form, Vol. 60 (2000), 93-121.

[9] R. DenK, M. HIeber, J. PrÜSs, R-boundedness, Fourier multipliers and problems of elliptic and parabolic type, Mem. Amer. Math. Soc., 166 (2003), n. 788.

[10] G. Da Prato And A. LunARdi, Solvability on the real line of a class of linear Voltera integro differential equations, Ann. Mat. Pura Appl., 55 (1988), 67-118.

[11] R. Haller, H. HeCK, A. Noll, Mikhlin's theorem for operator-valued Fourier multipliers in $n$ variables, Math. Nachr., 244 (2002), 110-130.

[12] HANs ENGLER, Strong solutions of quasiliniar integro-differential equations with singular kernels in several space dimension, Electronic Journal of Differential Equations, Vol. 1995 (1995), No. 02, pp. $1-16$.

[13] V. Keyantuo, Carlos Lizama, Maximal regularity for a class of integro-differential equations with infinite delay in Banach spaces, Studia Math., 168 (2005), 25-50.

[14] S. G. KREIN, Linear differential equations in Banach space, Providence, 1971.

[15] J. PRÜss, Evolutionary integral equations and applications, Birkhauser, Basel, 1993.

[16] V. PoBlete, Solutions of second-order integro-differential equations on periodic Besov spaces, Proceedings of the Edinburgh Mathematical Society, 50 (2007), 477-492. 
[17] P. E. Sobolevs KII, Inequalities coerciveness for abstract parabolic equations, Dokl. Akad. Nauk. SSSR, 57, 1 (1964), 27-40.

[18] V. B. Shakhmurov, Theorems about of compact embedding and applications, Doklady Akademii Nauk SSSR, 241, 6 (1978), 1285-1288.

[19] V. B. SHAKHMUROV, Coercive boundary value problems for regular degenerate differential-operator equations, J. Math. Anal. Appl., 292, 2 (2004), 605-620.

[20] V. B. SHAKHMUROV, Embedding theorems and maximal regular differential operator equations in Banach-valued function spaces, Journal of Inequalities and Applications, 2, 4 (2005), 329-345.

[21] V. B. Shakhmurov, Embedding and maximal regular differential operators in Banach-valued weighted spaces, Acta mathematica Sinica, 22, 5 (2006), 1493-1508.

[22] H. TRIEBEL, Interpolation theory. Function spaces. Differential operators., North-Holland, Amsterdam, 1978.

[23] V. VERGARA, Maximal regularity and global well-posedness for a phase field system with memory, Journal of Integral Equations and Applications, 19 (1), Spring 2007.

[24] L. WeIS, Operator-valued Fourier multiplier theorems and maximal $L_{p}$ regularity, Math. Ann., 319 (2001), 735-75?.

[25] S. Yakubov and Ya. Yakubov, Differential-operator equations. Ordinary and Partial Differential equations, Chapmen and Hall/CRC, Boca Raton, 2000. 